\title{
MEASURE AND MONITOR STOCK LEVELS
}

Henry Schein Minerva's eZscan is a time-saving innovation designed to enhance the sale of oral hygiene products, enabling any practice to easily generate additional revenue streams and giving patients the full benefit of professional recommendation.

This barcode reading device is capable of measuring and monitoring stock levels ensuring that merchandising units are kept well-stocked with the items you sell most. eZscan allows you to simply scan the barcodes of the products you would like to order, either from previous stock or by using the Henry Schein Minerva catalogue that is fully bar-coded for convenience. By connecting the scanner directly to your PC you can upload your completed list, making ordering quick, easy and preventing errors.

Readership response number 59

\section{CARE OF GUMS AND DENTURES}

Curaprox's Denture and Gum Care Set provides patients with products for the very best care of gums and dentures. The natural ingredients in Curaprox's weekly cleaning fluid and daily gel protects the denture composition, maintaining the original colour and finish of the acrylic resin, while the

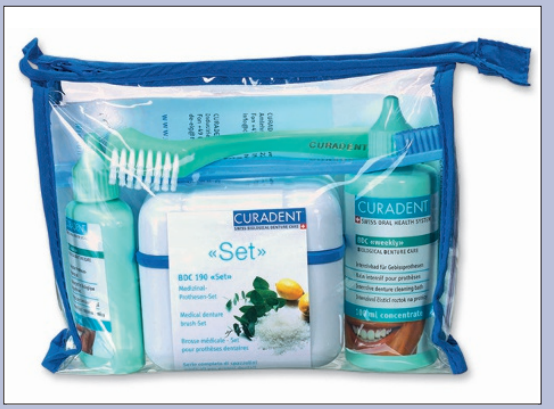

daily brushing and cleaning denture gel is gentle yet effective.

The Denture and Gum Care Set contains an ergonomically designed denture cleaning brush, a CS 5460 Ultra Soft Brush to massage and stimulate blood supply and enhance mucosa secretions for healthy gums, weekly denture bath concentrate containing citric acid for effective plaque removal and eucalyptus oil to form a microfilm on dentures, leaving a fresh taste and surface protection, daily brushing/cleaning denture gel and a neat, durable box suitable for storage, soaking or cleaning of dentures.

The set also includes an instruction leaflet so patients are aware of the importance of a gum and denture cleaning regimen to their oral health. Readership response number 60

\section{ORAL HEALTH INFORMATION SYSTEM}

Oralinsights from PracticeWorks is a portable, interactive oral health information system that enables both patient and dental professional to view a patient's tooth brushing behaviour on screen in real time 3D.

Generating a virtual image of a patient's dentition, the system can illustrate areas that require more attention when brushing. Use of educational feedback for both children and adolescents is simultaneously provided during the session in the form of red 'virtual plaque', which is gradually removed as the patient brushes using the correct technique.

Motivational feedback is produced in the form of an individual report, indicating what improvements could be made in which areas of the mouth for optimum oral hygiene technique.

Oralinsights' ease of use and interactive technology can develop children's motivation for brushing and assist in preventative care strategies. PracticeWorks offers both live and recorded online training for users, backed with customer support and aftercare.

Readership response number 61

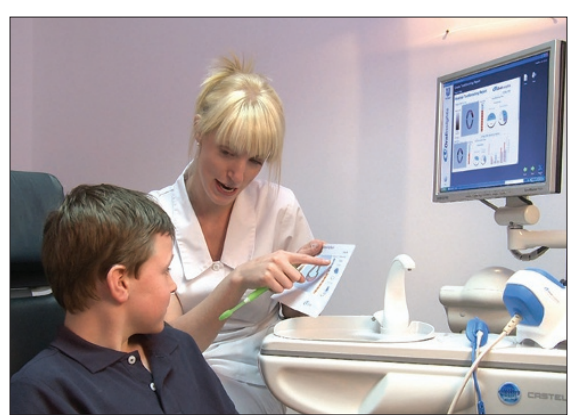

